\title{
Performance Limitations in Autocatalytic Networks in Biology ${ }^{\dagger}$
}

\author{
Nader Motee ${ }^{1}$, Fiona Chandra ${ }^{1}$, Bassam Bamieh $^{2}$, Mustafa Khammash ${ }^{2}$, and John C. Doyle ${ }^{1}$
}

\begin{abstract}
Autocatalytic networks, where a member can stimulate its own production, can be unstable when not controlled by feedback. Even when such networks are stabilized by regulating control feedbacks, they tend to exhibit non-minimum phase behavior. In this paper, we study the hard limits of the ideal performance of such networks and the hard limit of their minimum output energy. We consider a simplified model of glycolysis as our motivating example. For the glycolysis model, we characterize hard limits on the minimum output energy by analyzing the limiting behavior of the optimal cheap control problem for two different interconnection topologies. We show that some network interconnection topologies result in zero hard limits. Then, we develop necessary tools and concepts to extend our results to a general class of autocatalytic networks.
\end{abstract}

\section{INTRODUCTION}

Autocatalysis refers to any cyclical concatenation of processes wherein each member has the tendency to accelerate the activity of the preceding link and therefore stimulating its own production. This structure originates and stimulates growth of its constituent members until some physical or spatial constraint has been reached or a negative feedback stops the process of growth, allowing the system to settle in a stable state. Without such constraints or feedback, the autocatalytic system is inherently unstable. In metabolic systems the destabilizing effects of positive autocatalytic feedback is often countered by negative feedback loops, but high feedback gain can also move the system into a limit cycle.

Autocatalytic feedbacks commonly exhibit lags in time, and the time it takes one state variable to respond to changes in another may cause overshoot followed by an undershoot in the transient response. This is a typical behavior of a nonminimum phase system. This type of performance may be detrimental in live cells which in some cases may require more precision in both concentration and timing to function, yet autocatalytic networks are both ubiquitous and necessary in engineered and biological systems. Because of this, the operating point of such system is therefore constrained and the optimal performance we can gain from negative feedback

$\dagger$ The authors acknowledge research funding from the ONR through Grant N00014-08-1-0747, the ARO through Grant W911NF-08-1-0233, the AFOSR through Grants FA9550-08-1-0043 and FA9550-10-1-0143, the NSF through Grants EFRI-0735956 and ECCS-0835847 and ECCS0802008 and CMMI-0626170, the NIH through Grant R01-GM04983 and the Institute for Collaborative Biotechnologies through Grant DAAD19-03D-0004 from the US Army Research Office.

${ }^{1}$ The authors are with the Control and Dynamical Systems Department, California Institute of Technology, 1200 E. California Blvd, Pasadena, CA 91125 USA ( motee, fiona, doyle\}@cds.caltech.edu).

2 The authors are with the Department of Mechanical Engineering, University of California, Santa Barbara, CA 93106, USA ( $\{$ bamieh, khammash\}@engineering.ucsb.edu) . control is bounded. This bound exists in all systems and is typically even more aggravated in systems with autocatalysis.

With biology as our main motivation, first we look at the hard limits of a simplified glycolysis model given different optimal feedback mechanisms. For the glycolysis model, we consider optimal control problem with a quadratic cost function, in which a small parameter multiplies the control cost. This is the so called cheap control problem [1]. The key idea is the performance of an optimal control scheme is close to the ideal performance if the control effort is cheap and can be achieved if the control effort is free. This corresponds to solving an optimal control problem where the control effort is scaled by a small parameter $\epsilon>0$ and the ideal performance is calculated as $\epsilon \rightarrow 0$. This problem is important in analyzing the limiting possibilities of feedback controllers. One can used the result as a design principle in the form of high-gain feedback. The linear cheap control problem is well-studied [2]-[6].

In [7], a nonlinear cheap control analysis is proposed which highlights the role of unstable zero-dynamics- the nonlinear counterpart of nonminimum-phase (NMP) zeros. In Section III, we apply the result of [7] by looking at the zero-dynamics of the glycolysis model. We show that unstable zero-dynamics of the glycolysis model represents a structural obstacle to achieving zero ideal performance and that imposes a hard limit. This hard limit is exactly the least amount of output energy required to stabilize the zero-dynamics. We characterize this hard limit in terms of systems parameters and the strength of the autocatalytic feedback. The interconnection topology of the autocatalytic feedback and regulating control feedback in an autocatalytic network plays an important role in imposing hard limit on the minimum output energy. In the simplified glycolysis model, the autocatalytic and regulating control feedbacks are applied both on the same biochemical reaction. In fact, the unstable zero-dynamics in glycolysis model is an artifact of such interconnection topology.

Furthermore, we show how hard limits on the minimum output energy can disappear by altering the interconnection topology of the autocatalytic system. The topology of the glycolysis model is altered by applying autocatalytic and regulating control feedbacks on two different biochemical reactions. We investigate the limiting behavior of the optimal cheap control problem for the altered system by employing power series methods [9], [10]. The power series method was originally proposed to find numerical solution of HamiltonJacobi-Bellman (HJB) PDE that arises in an infinite-horizon (nonlinear) optimal control problem. We employ this method to find the solution of the optimal cheap control problem 
analytically. More specifically, we show that for the altered autocatalytic system the ideal performance is zero when the control effort is free. Therefore, the minimum output energy of the system can enjoy zero hard limit by altering the interconnection topology of the system in a specific way.

In Section IV, we introduce a class of large-scale autocatalytic networks. We characterize various types of autocatalytic feedbacks in an autocatalytic network. We develop underlying concepts and tools in order to extend our results from Section III to general autocatalytic networks. By applying concepts from graph theory, it is shown that under some sufficient conditions the zero-dynamics of an autocatalytic network is unstable. This motivates the analysis of the limiting behavior of the optimal cheap control problem for general autocatalytic networks.

\section{Two-State Regulated Autocatalytic Model OF GLYCOLYTIC PATHWAY}

Although autocatalytic networks can be unstable or have a constrained ideal performance, it is in some cases also unavoidable. Glycolysis is perhaps the most common control system on the planet and has been widely studied. Glycolysis generates ATP, which is the cell's energy currency. Similar to an engineered power plant whose machinery runs on its own energy product, the glycolysis reaction is autocatalytic and is therefore ideal to motivate theoretical analysis of biological systems. In the glycolysis pathway, autocatalysis of Adenosine triphosphate (ATP) is necessary for the steady state efficiency of this energy producing pathway. Glycolysis converts the sugar glucose into energy in the form of ATP, but initial investment of ATP molecules are necessary in the first steps of the pathway in order to convert glucose into something that would react more readily [13]. Two molecules of ATP are consumed in the early steps (hexokinase, phosphofructokinase/PFK) and four ATPs are generated as pyruvate is produced, hence producing an autocatalytic loop of ATP with a net gain of 2 molecules. We use glycolysis as our motivating example and look at a simplified model of glycolysis previously presented in [11]. Early experimental observations suggest that there are two Hopf modes present in the system and that a two-state model centered on an abstracted version of PFK is a reasonable simplification of glycolysis. We consider the two-state model from [11] with ATP (denoted by variable $y$ ) and a lumped intermediate metabolite (denoted by variable $x$ ) as states. We assume that the total concentration of adenosine phosphates in the cell remains constant, i.e., $[$ Atot $]=[\mathrm{ATP}]+[\mathrm{ADP}]+[\mathrm{AMP}]$, and hence the activating effects of AMP can be expressed as inhibition by ATP. We assume that the decay rates of the metabolites and the intermediate reaction converting $x$ to $y$ are operating in the linear regime and obey simple mass action kinetics:

$$
\begin{aligned}
& \dot{x}=-k_{x} x+\frac{V y^{q}}{1+\gamma y^{h}} \\
& \dot{y}=-k_{y} y+(1+q) k_{x} x-q \frac{V y^{q}}{1+\gamma y^{h}}
\end{aligned}
$$

in which $q$ is the number of $y$ molecules that are invested in the pathway, and $q+1$ is the number of $y$ molecules produced. The parameter $k_{x}$ represents the lumped metabolic reactions that generate ATP, and $h$ is the gain of the inhibition of the enzymes by ATP. The parameter $k_{y}$ represents the ATP demand of the cell, and we assumed that ATP-dependent processes are in saturation, and thus constant. In this case the glycolytic flux is set by the ATP demand of the cell or the reactor in the case of cell extract experiment. As a reminder, glycolytic oscillations have so far mainly been seen in anaerobic conditions, and so there is no additional ATP production from aerobic pathways. We normalize the model such that the system produces one more molecule of $y$ than the number consumed, i.e., consumes $q y$ molecules and produces $(1+q) y$ molecules.

For the sake of simplicity of notations, we assume that the equilibrium of interest is $\bar{y}=1$. This can be done by further non-dimensionalizing the model with respect to the concentration and flux of $y$. The equilibrium of interest can be characterized as

$$
\left[\begin{array}{l}
\bar{x} \\
\bar{y}
\end{array}\right]=\left[\begin{array}{c}
\frac{k_{y}}{k_{x}} \\
1
\end{array}\right] \quad \text { where } \quad k_{x} \bar{x}=k_{y}=\frac{V}{1+\gamma} .
$$

\section{IdeAl PERFormance of AutocatalytiC CONTROL SYSTEMS}

In the glycolytic pathway model (1)-(2), we can interpret $u=\frac{1}{1+\gamma y^{h}}$ as the regulatory feedback control that captures the inhibition of the catalyzing enzyme. In this section, we derive two control system models from the regulated autocatalytic model of the glycolytic pathway (1)-(2). First, we consider a network structure where autocatalytic and regulating control feedbacks are applied both on the same biochemical reaction (see Fig. 1). In the second scenario, we consider a system where autocatalytic and regulating control feedbacks are applied each on a different reaction (see Fig. 2 ). We show that the first network structures imposes a hard limit on the ideal performance of the system while the second network structure allows the system to achieve the ideal performance with cheap control strategy.

We consider the first scenario which corresponds to the following autocatalytic control system model

$$
\begin{aligned}
& \dot{x}=-k_{x} x+V y^{q} u \\
& \dot{y}=-k_{y} y+(q+1) k_{x} x-q V y^{q} u
\end{aligned}
$$

The linearization around the non-zero equilibrium point (3) yields an LTI system with the following state-space matrices

$$
A_{1}=\left[\begin{array}{cc}
-k_{x} & q k_{y} \\
(1+q) k_{x} & -\left(1+q^{2}\right) k_{y}
\end{array}\right], \quad B_{1}=\left[\begin{array}{c}
V \\
-q V
\end{array}\right]
$$

The transfer function from control input $u$ to output $y$ is given by

$$
G_{1}(s)=\frac{V\left(-q s+k_{x}\right)}{s^{2}+\left(k_{y}+k_{y} q^{2}+k_{x}\right) s-(q-1) k_{x} k_{y}}
$$

The linearized model has a zero in $\mathbb{C}_{+}$at $\frac{k_{x}}{q}$ and that it is nonminimum phase. One can also feature the nonminimum 


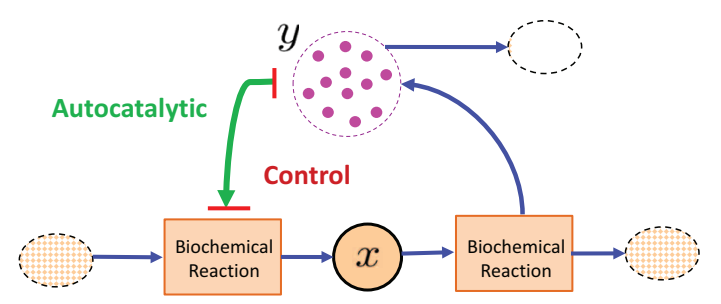

Fig. 1: A simple autocatalytic system where autocatalysis feedback and regulating control feedback are applied on the same biochemical reaction. This diagram corresponds to system (4)-(5).

phase nature of the system by introducing a new variable $z=x+\frac{1}{q} y$ and rewriting the system (4)-(5) in the following canonical form

$$
\begin{aligned}
& \dot{y}=-\left(k_{y}+\left(1+\frac{1}{q}\right) k_{x}\right) y+(q+1) k_{x} z-q V y^{q} u \\
& \dot{z}=\left(\frac{k_{x}}{q}\right) z-\left(\frac{k_{x}}{q^{2}}+\frac{k_{y}}{q}\right) y
\end{aligned}
$$

The subsystem (7) represents the zero-dynamics of (4)(5). The zero-dynamics (7) is antistable, i.e., $\dot{z}=-\left(\frac{k_{x}}{q}\right) z$ is asymptotically stable. One can think of $y$ as a control input to unstable subsystem (7). One can see that some minimum output energy (i.e., ATP) is required to stabilize unstable zero-dynamics (7). This output energy represents the energetic cost of the cell to stabilize to its steady state. The following theorem, we show that the minimum output energy is bounded from below by a constant which is only a function of parameters and initial condition of the glycolysis model. This hard limit is independent of of the feedback control strategy used to stabilize the system.

Theorem 1: Suppose that the equilibrium of interest is given by (3) and $\bar{u}=\frac{1}{1+\gamma \bar{y}^{h}}$. Consider the optimal cheap control problem for system (4)-(5) where the objective is to minimize cost functional

$$
J_{\epsilon}=\frac{1}{2} \int_{0}^{\infty}\left((y(t)-\bar{y})^{2}+\epsilon^{2}(u(t)-\bar{u})^{2}\right) d t
$$

where $\epsilon>0$ is sufficiently small. Then, the optimal cost value is

$$
J_{\epsilon}^{*}(x(0), y(0))=\frac{q^{3} k_{x}}{\left(q k_{y}+k_{x}\right)^{2}} \tilde{z}(0)^{2}+\mathcal{O}(\epsilon) .
$$

where

$$
\tilde{z}(0)=(x(0)-\bar{x})+\frac{1}{q}(y(0)-\bar{y}) \quad \text { and } \quad\left[\begin{array}{l}
x(0) \\
y(0)
\end{array}\right] \in \mathbb{R}_{+}^{2} .
$$

The ideal performance can be obtained from (9) as $\epsilon \rightarrow 0$. Furthermore, there is a hard limit on the total output energy of system (4)-(5), i.e.,

$$
\min _{u \in \mathcal{U}} \frac{1}{2} \int_{0}^{\infty}(y(t)-\bar{y})^{2} d t \geq J_{0}^{*}(x(0), y(0))
$$

where $\mathcal{U}$ is the set of all stabilizing state feedback control laws for system (4)-(5).

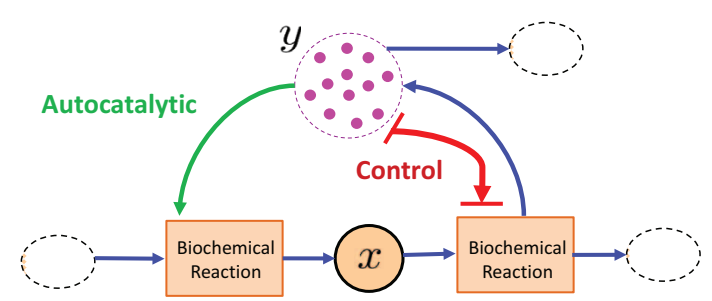

Fig. 2: A simple autocatalytic system where autocatalysis feedback and regulating control feedback are applied on different biochemical reaction. This diagram corresponds to system (13)-(14).

Proof: We refer to [14] for a proof.

We emphasize that the result of Theorem 1 asserts that structure of the autocatalytic network (as shown in Fig. 1) is the main reason for existence of a hard limit on the ideal performance. In the following, we show that this hard limit results in some performance limitations and tradeoffs. The zero-dynamics of the glycolysis model (4)-(5) is linear with the following transfer function

$$
Z(s)=-\frac{\frac{k_{x}}{q^{2}}+\frac{k_{y}}{q}}{s-\frac{k_{x}}{q}} Y(s)
$$

A simple performance requirement is to have small steadystate gain when there is a small constant disturbance in ATP consumption, i.e., $y \rightarrow y+\delta$. The steady-state value is given by

$$
z(\infty)=\left(\frac{1}{q}+\frac{k_{y}}{k_{x}}\right) \delta
$$

We assume that the equilibrium of the system remains fixed, i.e., $\bar{x}=\frac{k_{y}}{k_{x}}$. According to inequality (10), the glycolysis mechanism is more energy efficient if $q$ is small. On the other hand from (12), small autocatalysis $q$ deteriorates achievable steady-state disturbance rejection.

Remark 1: We note that inequality (10) is not tight. A more realistic scenario is to obtain a hard limit by optimizing the output energy over all output (rather than full-state) feedback control laws. This is usually the case in biological systems. For example in Glycolysis model (1)-(2), output feedback control law $u(y)=\frac{1}{1+\gamma y^{h}}$ stabilizes the process. However, adding this new constraint to our problem makes the analysis quite difficult.

In most biological experiments, it is often the case that the equilibrium of the process is attained in $T<\infty$ seconds. In such situations, the inequality (10) reduces to

$$
\sup _{t \in[0, T]}|y(t)-\bar{y}| \geq \sqrt{\frac{2 J_{0}^{*}(x(0), y(0))}{T}}
$$

This inequality provide a lower bound on the peak error for a given reference step input $\bar{y}$ and a given settling time $T$. According to Theorem 1, this hard limit is dictated by the unstable zero-dynamics of (4)-(5).

Next, we show that if one modifies the structure of network in (4)-(5) so that autocatalysis and regulating control 
feedbacks are applied on different biochemical reactions (as shown in Fig. 2), one can achieve the ideal performance, i.e., zero hard limits on the total output energy. To this end, we consider the following autocatalytic system

$$
\begin{aligned}
& \dot{x}=-k_{x} x u+V y^{q} \\
& \dot{y}=-k_{y} y+(q+1) k_{x} x u-q V y^{q} .
\end{aligned}
$$

The linearization around the non-zero equilibrium point (3) yields an LTI system with the following state-space matrices

$$
A_{2}=\left[\begin{array}{cc}
-\frac{k_{x} k_{y}}{V} & q V \\
\frac{(1+q) k_{x} k_{y}}{V} & -k_{y}-q^{2} V
\end{array}\right], \quad B_{2}=\left[\begin{array}{c}
-k_{y} \\
(1+q) k_{y}
\end{array}\right]
$$

It is straightforward to verify that the transfer function from input $u$ to output $y$ is

$$
G_{2}(s)=\frac{(1+q) k_{y} s}{s^{2}+\left(k_{y}+V q^{2}+\frac{k_{x} k_{y}}{V}\right) s+\frac{k_{x} k_{y}^{2}}{V}-q k_{x} k_{y}}
$$

The linearized model has a zero at the origin and therefore it is nonminimum phase. This is clearly due to the particular autocatalytic network structure. Despite the fact the linearized model has a zero at the origin, in the next theorem we show that there is no hard limits on the ideal performance of the nonlinear system (13)-(14).

Theorem 2: Suppose that the equilibrium of interest is given by (3) and $\bar{u}=1$. Consider optimal cheap control problem for system (13)-(14) where the objective is to minimize cost functional (8) for sufficiently small $\epsilon>0$. Then, the optimal cost value is

$$
J_{\epsilon}^{*}(x(0), y(0))=\mathcal{O}(\epsilon) .
$$

Furthermore, the ideal performance can be obtained as $\epsilon \rightarrow 0$ and therefore

$$
\min _{u \in \mathcal{U}} \frac{1}{2} \int_{0}^{\infty}(y(t)-\bar{y})^{2} d t \geq 0
$$

where $\mathcal{U}$ is the set of all stabilizing state feedback control laws for system (13)-(14).

\section{Proof: We refer to [14] for a proof.}

\section{General Autocatalytic Networks}

We have just analyzed one example of autocatalytic networks as represented by glycolysis. Both in engineering and in biology, we find many other autocatalytic networks with varied topology. For example, the blood clotting pathway is composed of a nested series of autocatalytic loops [12]. In this section, we will generalize our results for general autocatalytic networks. An autocatalytic set is a collection of entities, each of which can be created catalytically by other entities within the set, such that as a whole, the set is able to catalyze its own production. To some extent, we can formalize the autocatalysis concept using graph theoretical tools. We can imagine a given set of biochemical reactions as a directed graph $\mathcal{G}$ (reaction graph) in which a node represents a molecular species and an edge from node $j$ to node $i$ means that $j$ is a catalyst for $i$. From a control

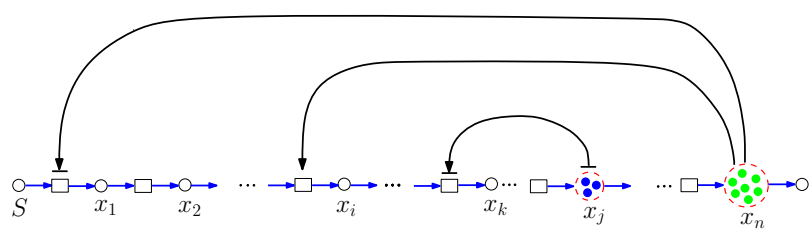

Fig. 3: A schematic graph of an autocatalytic network. Each square box represents a biochemical reaction and each circle denotes the byproduct of the biochemical reaction. In this network, autocatalytic feedback $\mathcal{A}_{n i}$ is of type I, $\mathcal{A}_{\bar{j} \bar{k}}$ of type II, and $\mathcal{A}_{n \overline{1}}$ of type III.

theoretic perspective, each edge can be interpreted as a catalytic and regulating control feedback loop (see Fig. 3 and Table I).

Definition 1: A subgraph $\mathcal{G}_{a}$ of a reaction graph $\mathcal{G}$ is called autocatalytic if every node of $\mathcal{G}_{a}$ has at least one incoming edge from another node of $\mathcal{G}_{a}$.

In general, autocatalytic subgraphs are different from cycles and irreducible subgraphs. In fact, there is a hierarchical inclusion relationship between cycles, irreducible subgraphs, and autocatalytic subgraphs as follows

cycles $\subset$ irreducible subgraphs $\subset$ autocatalytic subgraphs.

We define a class of autocatalytic networks with multiple autocatalytic and regulating control feedbacks (see Figure 3). Roughly speaking, an autocatalytic feedback can be thought of as an energy delivery channel from a source node to a sink node. In an autocatalytic feedback from node $j$ to node $i$ (denoted by $\mathcal{A}$ ), byproduct of biochemical reaction $j$ (denoted by $x_{j}$ ) catalyzes the evolution of species $i$ in order to help to create more $x_{j}$. In this case, $j$ is the source node and $i$ the sink node of the autocatalysis feedback. We denote $j=\operatorname{source}\{\mathcal{A}\}$ and $i=\operatorname{sink}\{\mathcal{A}\}$. For an autocatalytic network with underlying autocatalytic graph $\mathcal{G}$, the set of all autocatalytic feedbacks is represented by $\operatorname{ACL}(\mathcal{G})$.

Now we have enough machinery to characterize an inetersting class of autocatalytic networks. An autocatalytic network consists of a collection of interconnected subsystems each with the following dynamics

$$
\dot{x}_{i}=-g_{i}\left(x_{i}\right)+w_{i}
$$

for $i=1, \ldots, n$. It is assumed that functions $g_{i}$ are differentiable and increasing functions and $g_{i}(0)=0$. The network interconnection topology is determined from the underlying autocatalytic graph of the network. In Table I, we characterize various types of autocatalysis feedbacks based on their role in catalyzing and controlling the corresponding biochemical reactions. For example, in a type III autocatalytic feedback, only one of the biochemical reactions of the end nodes (source or sink) is regulated by a control input. In Fig. 3 shows a type III autocatalytic feedback from node $n$ to 1 with a regulating control input on biochemical reaction 1 which is denoted by $\mathcal{A}_{n \overline{1}}$.

Without loss of generality, we may assume that the byproduct of the first reaction does not catalyze any of 


\begin{tabular}{|c|c|c|c|}
\hline Type & Regulating Control Input & Notation & Schematic \\
\hline I & None & $\mathcal{A}_{j i}$ & $\longleftarrow$ \\
\hline II & Source and Sink & $\mathcal{A}_{\bar{j} \bar{i}}$ & $\longleftarrow$ \\
\hline III & Either Source or Sink & $\mathcal{A}_{\bar{j} i}$ or $\mathcal{A}_{j \bar{i}}$ & $\longleftarrow$ or $\longleftarrow$ \\
\hline
\end{tabular}

TABLE I: Possible control scenarios for an autocatalysis feedback from subsystem $j$ to subsystem $i$.

the biochemical reactions in the network. Based on this assumption and our notations, $w_{1}=0$ if subsystem 1 is not catalyzed, otherwise

$$
w_{1}=\sum_{j}\left\{\begin{array}{ccc}
\kappa_{j 1}\left(x_{j}\right) u_{j 1} & \text { if } & 1 \in \operatorname{sink}\left\{\mathcal{A}_{j \overline{1}}\right\} \\
\kappa_{j 1}\left(x_{j}\right) & \text { if } & 1 \in \operatorname{sink}\left\{\mathcal{A}_{j 1}\right\}
\end{array}\right.
$$

where $\kappa_{j i}$ captures the effect of autocatalytic feedback on biochemical reaction 1 . The interconnection topology of the rest of the network can be modeled according to the underlying autocatalytic graph of the entire system as follows. For $i=2, \ldots, n$ and depending on the structure of the underlying autocatalytic graph, we define:

- If $i$ is not a sink or source node:

$$
w_{i}=g_{i-1}\left(x_{i-1}\right)
$$

- If $i=\operatorname{sink}\left\{\mathcal{A}_{j *}\right\}$ :

$$
w_{i}=\sum_{j} \begin{cases}g_{i-1}\left(x_{i-1}\right) \kappa_{j i}\left(x_{j}\right) & \text { if } i \in \operatorname{sink}\left\{\mathcal{A}_{j i}\right\} \\ g_{i-1}\left(x_{i-1}\right) \kappa_{j i}\left(x_{j}\right) u_{j i} & \text { if } i \in \operatorname{sink}\left\{\mathcal{A}_{j i}\right\}\end{cases}
$$

- If $i \in \operatorname{source}\left\{\mathcal{A}_{* j}\right\}$ :

$$
\begin{aligned}
w_{i}=f_{i-1}\left(x_{i-1}\right)- \begin{cases}\eta_{i 1}\left(x_{i}\right) & \text { if } i \in \operatorname{source}\left\{\mathcal{A}_{i 1}\right\} \\
\eta_{i 1}\left(x_{i}\right) u_{i 1} & \text { if } i \in \operatorname{source}\left\{\mathcal{A}_{\overline{1}}\right\}\end{cases} \\
-\sum_{j} \begin{cases}g_{j-1}\left(x_{j-1}\right) \eta_{i j}\left(x_{i}\right) & \text { if } i \in \operatorname{source}\left\{\mathcal{A}_{i j}\right\} \\
g_{j-1}\left(x_{j-1}\right) \eta_{i j}\left(x_{i}\right) u_{i j} & \text { if } i \in \operatorname{source}\left\{\mathcal{A}_{\bar{i} j}\right\}\end{cases}
\end{aligned}
$$

The output vector is defined as

$$
y_{k}=x_{j_{k}}, \quad k=1, \ldots, M
$$

where $j_{k}=\operatorname{source}\{\mathcal{A}\}$ for all $\mathcal{A} \in \operatorname{ACL}(\mathcal{G})$ and $M$ is the number of autocatalytic feedbacks in the network. The functions $\kappa_{j i}, \eta_{j i}$, and $f_{j}$ are differentiable and increasing functions and $\kappa_{j i}(0)=\eta_{j i}(0)=f_{j}(0)=0$. The function $\kappa_{j i}$ capture the effect of the autocatalysis feedback from node $j$ to $i$ on the corresponding biochemical reactions.

Assumption 1: For an autocatalytic feedback from node $j$ to $i$, we assume that

$$
\kappa_{j i}(x)<\eta_{j i}(x) \quad \text { and } \quad g_{j}(x)<f_{j}(x)
$$

for all $x \in \mathbb{R}_{+}$.

Lemma 1: Consider autocatalytic network defined by (17)-(22) with no autocatalytic feedback. Then the origin $x=\mathbf{0}$ is a globally asymptotically stable equilibrium of the system for all $x(0) \in \mathbb{R}_{+}^{n}$.

Proof: When there is no autocatalytic feedback, the dynamics of the network is governed by the following differential equations

$$
\begin{aligned}
& \dot{x}_{1}=-g_{1}\left(x_{1}\right) \\
& \dot{x}_{i}=-g_{i}\left(x_{i}\right)+g_{i-1}\left(x_{i-1}\right)
\end{aligned}
$$

for $i=2, \ldots, n$. From our assumptions that $g_{i}(0)=0$ it follows that the unique equilibrium of (23)-(24) is $x^{*}=\mathbf{0}$. The Jacobian matrix evaluated at the origin is given by

$$
J(\mathbf{0})=\left[\begin{array}{ccccc}
-g_{1}^{\prime}(0) & 0 & 0 & \cdots & 0 \\
g_{1}^{\prime}(0) & -g_{2}^{\prime}(0) & 0 & \cdots & 0 \\
\vdots & & \ddots & & \vdots \\
0 & 0 & \cdots & -g_{n-1}^{\prime}(0) & 0 \\
0 & 0 & \cdots & g_{n-1}^{\prime}(0) & -g_{n}^{\prime}(0)
\end{array}\right] .
$$

Since all functions $g_{i}$ are increasing, it follows that $J(\mathbf{0})$ is Hurwitz. Therefore, $x=\mathbf{0}$ is a globally asymptotically stable equilibrium of the system for all initial condition in $\mathbb{R}_{+}^{n}$.

In the following theorem, we show that under some conditions that are imposed due to the existence of autocatalytic feedbacks in the network, the zero-dynamics of the network is unstable. This result helps to extend the results of Theorem 1 to arbitrary autocatalytic networks.

Definition 2: An autocatalytic network is simple if every node is either a source or sink of at most one autocatalytic feedback.

In this paper, we only present our results for simple autocatalytic networks with type II autocatalytic feedbacks. However, similar results hold for a general autocatalytic network defined by (17)-(22).

Theorem 3: For a simple autocatalytic network (17)-(22) with $M$ autocatalytic feedbacks of type II, suppose that $\bar{x} \in$ $\mathbb{R}_{+}^{n}$ is a desired equilibrium. Suppose that

$$
\frac{f_{j-1}^{\prime}\left(\bar{x}_{j-1}\right)}{g_{j-1}^{\prime}\left(\bar{x}_{j-1}\right)}>\frac{\eta_{j i}\left(\bar{x}_{j}\right)}{\kappa_{j i}\left(\bar{x}_{j}\right)}
$$

in which $j=\operatorname{source}\{\mathcal{A}\}$ and $i=\operatorname{sink}\{\mathcal{A}\}$ for all $\mathcal{A} \in \operatorname{ACL}(\mathcal{G})$. Then the zero-dynamics of the autocatalytic network is unstable.

Proof: Without loss of generality, we may assume that there is an autocatalytic feedback from node $n$ to 1 . In order to obtain the zero-dynamics of (17)-(22) at equilibrium point $\bar{x}$, we should impose the following constraints on the outputs of the system

$$
y_{k}-\bar{x}_{j_{k}}=x_{j_{k}}-\bar{x}_{j_{k}} \equiv 0
$$

where $j_{k}=\operatorname{source}\{\mathcal{A}\}$ for all $\mathcal{A} \in \operatorname{ACL}(\mathcal{G})$. By imposing these constraints and solving the resulting equations for control inputs, one can derive the zero-dynamics of (17)-(22) 
as follows

$$
\begin{aligned}
\dot{x}_{1} & =-g_{1}\left(x_{1}\right)+\frac{\kappa_{n, 1}\left(\bar{x}_{n}\right)}{\eta_{n, 1}\left(\bar{x}_{n}\right)}\left(f_{n-1}\left(x_{n-1}\right)-g_{n}\left(\bar{x}_{n}\right)\right) \\
\dot{x}_{2} & =-g_{2}\left(x_{2}\right)+g_{1}\left(x_{1}\right) \\
& \vdots \\
\dot{x}_{i} & =-g_{i}\left(x_{i}\right)+\frac{\kappa_{j i}\left(\bar{x}_{j}\right)}{\eta_{j i}\left(\bar{x}_{j}\right)}\left(f_{j-1}\left(x_{j-1}\right)-g_{j}\left(\bar{x}_{j}\right)\right) \\
& \vdots \\
\dot{x}_{n-1} & =-g_{n-1}\left(x_{n-1}\right)+g_{n-2}\left(x_{n-2}\right)
\end{aligned}
$$

In the following, we characterize conditions under which the zero-dynamics is unstable. The corresponding Jacobian matrix at $\bar{x}$ is given by

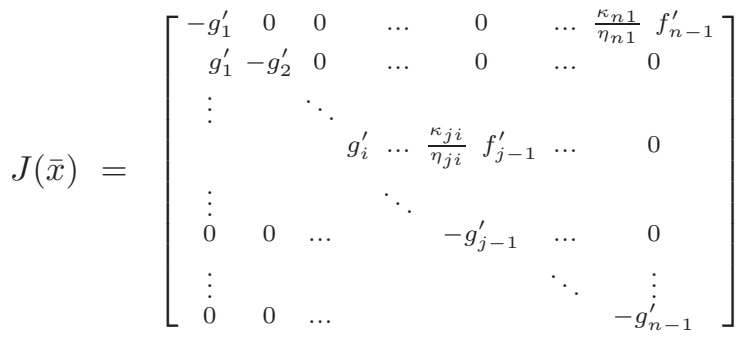

We can rewrite the Jacobian matrix in the following form

$$
J(\bar{x})=(-I+A(\bar{x})) D(\bar{x})
$$

in which

$$
A(\bar{x})=\left[\begin{array}{cccccccc}
0 & 0 & 0 & \ldots & 0 & \ldots & \frac{\kappa_{n 1}}{\eta_{n 1}} & \frac{f_{n-1}^{\prime}}{g_{n-1}^{\prime}} \\
1 & 0 & 0 & \ldots & 0 & \ldots & 0 \\
\vdots & \ddots & & & & \\
& & 1 & \ldots & \frac{\kappa_{j i}}{\eta_{j i}} & \frac{f_{j-1}^{\prime}}{g_{j-1}^{\prime}} & \cdots & 0 \\
\vdots & & & \ddots & & & \\
0 & 0 & \ldots & & 0 & \ldots & 0 \\
\vdots & & & & & \ddots & \vdots \\
0 & 0 & \ldots & & & & 0
\end{array}\right]
$$

and $D(\bar{x})=\operatorname{diag}\left(g_{1}^{\prime}\left(\bar{x}_{1}\right), \ldots, g_{n-1}^{\prime}\left(\bar{x}_{n-1}\right)\right)$. We can think of matrix $A(\bar{x})$ as adjacency matrix of a weighted graph. According to the proven lemmas in Appendix section of [14], if

$$
\frac{\kappa_{j i}\left(\bar{x}_{n}\right)}{\eta_{j i}\left(\bar{x}_{n}\right)} \frac{f_{j-1}^{\prime}\left(\bar{x}_{j-1}\right)}{g_{j-1}^{\prime}\left(\bar{x}_{j-1}\right)}>1,
$$

then $\lambda_{\max }(A(\bar{x}))>1$. Since $D(\bar{x})$ a positive diagonal matrix, if the above inequality holds, it concludes that $\lambda_{\max }(J(\bar{x}))>0$. Thus, the zero-dynamics of the autocatalytic network is unstable.

In the following corollary, we show that for an autocatalytic network with only one autocatalytic feedback of type II, one can explicitly characterize the zero of the transfer function of the linearized model of the network.

Corollary 1: Suppose that system (17)-(22) has only one autocatalysis feedback $\mathcal{A}_{\bar{n} \overline{1}}$ and

$$
f_{n-1}(x)=(a+b) g_{n-1}(x) \text { and } \eta_{n 1}(x)=a \kappa_{n 1}(x)
$$

for some given $a, b>0$ and all $x \in \mathbb{R}_{+}$. If we assume $g_{i}^{\prime}\left(\bar{x}_{i}\right)=1$ for all $i=1, \ldots, n-1$, then the poles of the linearization of the zero-dynamics at $\bar{x}$ are given by

$$
z_{k}=\left(-1+\sqrt[n-1]{1+\frac{b}{a}} \cos \frac{2 k \pi}{n-1}\right)+i \sin \frac{2 k \pi}{n-1} .
$$

Proof: We refer to [14] for a proof.

\section{CONCLUSION}

We have studied the ideal performance limitations of autocatalytic systems. We used a simplified model of the glycolysis pathway as a motivating example and characterized the performance limitations of the model. The optimal cheap control problem was considered for the glycolysis model and we analyzed the limiting behavior of this cheap control problem. We showed that the unstable zero-dynamics of the glycolysis model represents a structural obstacle to achieving zero ideal performance and therefore imposes a hard limit. This hard limit is exactly the least amount of output energy required to stabilize the zero-dynamics. We proved that for some interconnection topologies there is a hard limit on the ideal performance and we further show that by altering the interconnection topology in a specific way, the ideal performance can be achieved when the control effort is free. Then, we developed a mathematical foundation to study performance limitations for a class of autocatalytic networks. We formally defined a class of autocatalytic networks and showed that the zero-dynamics of such networks are unstable.

\section{REFERENCES}

[1] H. Kwakernaak and R. Sivan. "The maximally achievable accuracy of linear optimal regulators and Linear Optimal Filters," IEEE Transactions on Automatic Control, Vol. AC-17, pp. 79-85, 1972.

[2] B. Francis. "The optimal linear-quadratic time-invariant regulator with cheap control," IEEE Transactions on Automatic Control, Vol. AC-24, pp. 616-621, 1979.

[3] J. Grasman. "On a class of optimal control problems with an almost cost-free solution," IEEE Transactions on Automatic Control, Vol. AC27, pp. 441-445, 1982.

[4] R. H. Middleton. "Trade-offs in linear control system design," Automatica, Vol. 27, no. 2, pp. 281-292, 1991.

[5] L. Qiu and E. J. Davison. "Performance limitations of non-minimum phase systems in the servomechanism problem," Automatica, Vol. 29, no. 2, pp. 337-349. 1993.

[6] M. M. Seron, J. H. Braslavsky, and G. C. Goodwin. "Fundamental limitations in filtering and control," New York: Springer, Vol. 1997.

[7] M. M. Seron, J. H. Braslavsky, P. V. Kokotovic, and D. Q. Mayne. "Feedback limitations in nonlinear systems: from Bode integrals to cheap control," IEEE Transactions on Automatic Control, Vol. 44, no. 4, pp. 829-833. Apr. 1999.

[8] P. Sannuti and H. S. Wason. "Multiple time-scale decomposition in cheap control problems- singular control," IEEE Transactions on Automatic Control, Vol. 30, no7, pp. 633-644, 1985.

[9] E. G. Al'brecht. "On the optimal stabilization of nonlinear systems," Journal of Applied Mathematics and Mechanics,, Vol. 25, pp. 12541266, 1961.

[10] D. L. Lukes. "Optimal Regulation of Nonlinear Dynamical Systems," SIAM Journal on Control, Vol. 7, pp. 75-100, 1969.

[11] F. A. Chandra, G. Buzi, and J. C. Doyle. "Linear control analysis of the autocatalytic glycolysis system," In Proc. of the American Control Conference, 2009.

[12] J. Jesty, E. Beltrami. "Positive feedbacks of coagulation: their role in threshold regulation,"Arterioscler Thromb Vasc Biol, Vol 25, pp. 2463-9, 2005.

[13] J. M. Berg, J. L. Tymoczko, L. Stryer. Biochemistry. San Francisco: W. H. Freeman and Co., 2007.

[14] N. Motee, B. Bamieh, M. Khammash, J.C. Doyle. "Performance limitations in autocatalytic networks in biology," Technical Notes. Online: http://http://www.cds.caltech.edu/ motee/ publications.html 